\title{
Predicting reaction observables from back-scattering measurements in low- energy heavy-ion collisions
}

\author{
A. Diaz-Torres ${ }^{1}$, V.V. Sargsyan ${ }^{2,3}$, G.G. Adamian², N.V. Antonenko², P.R.S. Gomes ${ }^{4}$, and H. Lenske ${ }^{5}$ \\ ${ }^{1}$ European Centre for Theoretical Studies in Nuclear Physics and Related Areas, 38123 Villazzano, Trento, Italy \\ ${ }^{2}$ Joint Institute for Nuclear Research, 141980 Dubna, Russia \\ ${ }^{3}$ Yerevan State University, M. Manougian 1, 0025, Yerevan, Armenia \\ ${ }^{4}$ Instituto de Fisica, Universidade Federal Fluminense, Av. Litorânea, s/n, Niterói, R.J. 24210-340, Brazil \\ ${ }^{5}$ Institut für Theoretische Physik der Justus-Liebig-Universität, 35392 Giessen, Germany
}

\begin{abstract}
A simplified, reliable and useful method, based on reaction theory, for calculating a number of integrated and differential cross sections in low-energy heavy-ion collisions is presented. Simplified formulae provide predictions of reaction, capture and elastic-scattering differential cross sections, using experimental information about elastic and quasi-elastic back-scattering excitation functions.
\end{abstract}

\section{Introduction}

Nuclear reactions constitute the engine of stars and control nucleosynthesis. They are the key tool for extracting the properties of exotic nuclei at rare-isotope beam facilities. The physics of low-energy nuclear reactions is exciting as it integrates many-body nuclear structure, reaction dynamics and mechanisms as well as quantum mechanics. There is clear experimental evidence that nuclear structure and reaction dynamics are strongly coupled, the coupling determining the reaction outcomes [1]. To understand how two complex nuclei collide and to quantify the reaction observables are crucial objectives in this field.

The present contribution addresses a simplified, reliable method for calculating a number of integrated and differential cross sections, using (as input) experimental information on elastic and quasi-elastic excitation functions at 180 degrees [2]. Section 2 presents energy-shifting formulae which provide reliable capture and reaction probabilities [3], while in Sec. 3 these formulae are used in combination with information from back-scattering measurements for making reliable predictions of various cross sections. The reliability of the method is justified by the good agreement of its predictions with coupled channel calculations $[4,5]$. Conclusions are drawn in Sec. 4.

\section{Energy-shifting formulae}

Figure 1 illustrates the main idea of the energy-shifting formulae of the reaction and capture probabilities, explained in Ref. [3]. It consists in replacing the exact, reaction and capture probabilities for a nonzero angular momentum $J$ and bombarding energy $E$, with the corresponding s-wave probabilities evaluated at a lower energy, $\varepsilon_{J}=E-E_{r o t}(J)$. The rotational energy, $E_{r o t}(J)$, can be calculated in two different ways, as shown in Fig. 1. The first way is based on an expansion of the Coulomb barrier height, $V_{B}(J)$, up to second order in $\Lambda=J(J+1)$, and substracting the s-wave potential barrier, $V_{b}(0)$. The second way relies on a nuclear-modified Rutherford trajectory for the near-barrier projectile-target orbit. In these formulae, $\mu$ is the reduces mass, $R_{b}$ and $\omega_{b}$ are respectively the radius and curvature of the s-wave Coulomb barrier, while $\eta^{\prime}=Z^{\prime} \sqrt{\mu / 2 \hbar^{2} E}$ is an effective Sommerfeld parameter that includes the nuclear part of the nucleus-nucleus interaction potential [2]. The quantity $Z^{\prime}=Z_{P} Z_{T} e^{2}\left(1-a_{0} / R_{B}\right)$ [2], where $Z_{i}$ and $a_{0}$ refer to the charge number of the projectile and target nuclei and the diffuseness parameter of their bare nuclear interaction, respectively.

\section{Energy-Shifting Formulae}

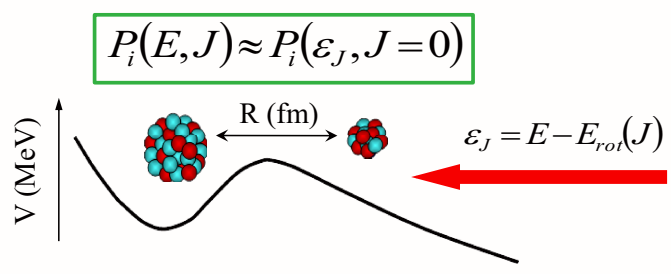

* Shifting Formula 1

$$
E_{r o t}(J)=\frac{\hbar^{2} \Lambda}{2 \mu R_{b}^{2}}+\frac{\hbar^{4} \Lambda^{2}}{2 \mu^{3} \omega_{b}^{2} R_{b}^{6}} \quad \Lambda=J(J+1)
$$

* Shifting Formula 2

$$
E_{r o t}(J)=E \frac{\left(\eta^{2}+J^{2}\right)^{1 / 2}-\eta^{\prime}}{\left(\eta^{2}+J^{2}\right)^{1 / 2}+\eta^{\prime}} \quad J=\eta^{\prime} \cot (\theta / 2)
$$

Figure 1. (Color online) Two energy-shifting formulae for capture and reaction probabilities. 
We have tested the validity of these formulae by carrying out coupled-reaction-channels (CRC) calculations [4, 5] for several heavy-ion systems, as presented in Ref. [3]. For instance, the partial reaction probabilities result from the elastic $S$-matrix elements: $P_{R}(E, J)=1-\left|S_{J}(E)\right|^{2}$. Please note that $P_{R}(E, J=0)$ and $P_{\text {cap }}(E, J=0)$ include CRC effects, such as long-range Coulomb excitations.

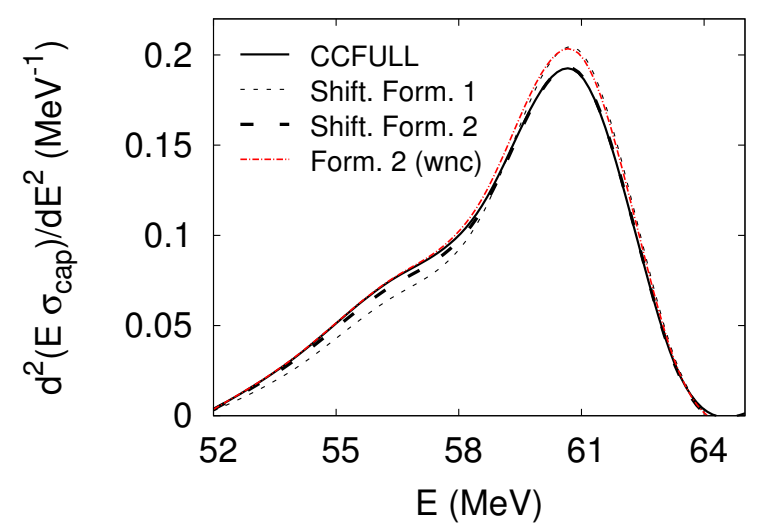

Figure 2. (Color online) Capture barrier distribution for ${ }^{16} \mathrm{O}$ $+{ }^{154} \mathrm{Sm}$. The exact coupled-channels outcomes are compared to those of the energy-shifting formulae, the barrier-distribution areas being normalized to unity. The agreement confirms the quality of the energy-shifting formulae, which is better for the second formula including the nuclear correction through the effective Sommerfeld parameter (dashed line). See Ref. [3] for details.

The agreement between the formula outcomes and the exact values are very good. The exact results are between those of the formulae, the second formula working a bit better than the first formula. The formula results improves notably, as the total mass $A=A_{T}+A_{P}$ increases and/or the mass asymmetry $\left[\eta=\left(A_{T}-A_{P}\right) / A\right]$ decreases. These two variables play an important role in the quality of the agreement as they impact on the moment of inertia of the dinuclear system. For instance, considering the moment of inertia $I=\mu R_{B}^{2}$, where $\mu \sim A\left(1-\eta^{2}\right) / 4$ and $R_{B} \sim A^{1 / 3}\left[(1-\eta)^{1 / 3}+(1+\eta)^{1 / 3}\right]$, the moment of inertia will be $I \sim A^{5 / 3}\left(1-\eta^{2}\right)\left[(1-\eta)^{1 / 3}+(1+\eta)^{1 / 3}\right]^{2}$. Consequently, $I$ rises with increasing $A$ and decreasing $\eta$, and the role of the centrifugal potential declines. It reduces the increasing rate of the rotational energy $E_{r o t}(J)$ as $J$ changes. Also the difference between $R_{B}$ and the distance of closest approach becomes smaller, so the two formulae in Fig. 1 provide similar rotational energies. Small $J$ values $(J \leq 30-40)$ are the main contributors to the heavy-ion capture and reaction cross sections at energies around the Coulomb barrier. The energy-shifting formulae are reliable in these collisions.

As an example, Fig. 2 shows the exact capture-barrier distribution and those of the energy-shifting formulae, for ${ }^{16} \mathrm{O}+{ }^{154} \mathrm{Sm}$. The nuclear correction (through the effective Sommerfeld parameter) appears to be crucial for the excellent performance of the second formula in Fig. 2 [compar-
Using the parameters of the $\mathrm{J}=\mathbf{0}$ Coulomb barrier and

$$
\begin{aligned}
& \text { * the Energy-Shifting Formula } 1 \\
& \sigma_{R}(E)=\frac{\pi R_{b}^{2}}{E} \int_{0}^{E} d \varepsilon P_{R}(\varepsilon, 0)\left[1-\frac{4(E-\varepsilon)}{\mu \omega_{b}^{2} R_{b}^{2}}\right] \\
& \sigma_{c a p}(E)=\frac{\pi R_{b}^{2}}{E} \int_{\varepsilon_{c r}}^{E} d \varepsilon P_{c a p}(\varepsilon, 0)\left[1-\frac{4(E-\varepsilon)}{\mu \omega_{b}^{2} R_{b}^{2}}\right] \\
& * \text { the Energy-Shifting Formula } 2 \\
& \sigma_{R}(E)=\frac{\pi Z^{\prime 2}}{E} \int_{0}^{E} d \varepsilon \frac{2 E-\varepsilon}{\varepsilon^{3}} \underline{P_{R}(\varepsilon, 0)} \\
& \sigma_{c a p}(E)=\frac{\pi Z^{\prime 2}}{E} \int_{\varepsilon_{J_{c r}}}^{E} d \varepsilon \frac{2 E-\varepsilon}{\varepsilon^{3}} \underline{P_{c a p}(\varepsilon, 0)} \quad Z^{\prime}=Z_{1} Z_{2} e^{2}\left(1-\frac{a_{0}}{R_{b}}\right)
\end{aligned}
$$

Figure 3. (Color online) Simplified formulae for reaction and capture cross sections. See Ref. [2] for details.

$$
\begin{aligned}
& P_{R}^{e x}(E, 0)=1-P_{e l}^{e x}(E, 0) \\
& P_{c a p}^{e x}(E, 0)=1-\left[P_{q e}^{e x}(E, 0)+P_{B U}^{e x}(E, 0)+P_{D I d}^{e x}(E, 0)\right]
\end{aligned}
$$

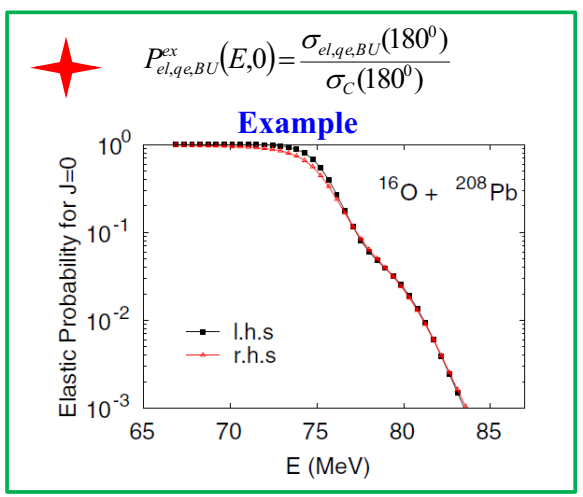

Figure 4. (Color online) Linking the simplified formulae with back-scattering measurements. See Ref. [2] for details.

ing the dashed line with the dash-dotted line that does not include the nuclear correction (wnc)].

\section{Simplified formulae for integrated and differential cross sections}

Figure 3 presents simplified formulae for reaction and capture cross sections [2]. These formulae stem from the exact expressions in reaction theory, replacing the discrete sum over angular momenta $J$ with an integral over $\varepsilon_{J}$ (see Fig. 1) and using the energy-shifting formulae for the reaction and capture probabilities.

These simplified expressions also provide accurate results for the reaction and capture cross sections, as demonstrated in Ref. [2]. Back-scattering measurements of elastic and quasi-elastic excitation functions can feed the simplified formulae, allowing the prediction of unknown integrated and differential cross sections. The main idea is for instance to identify the $P_{R}(E, J=0)$ and $P_{c a p}(E, J=0)$ 

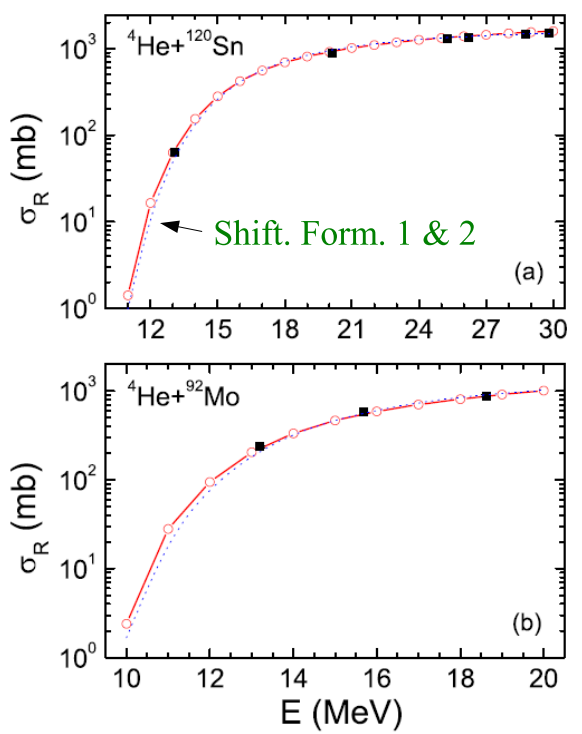

Figure 5. (Color online) Reaction cross sections from the simplified formulae compared with experimental data. See Ref. [2] for details.
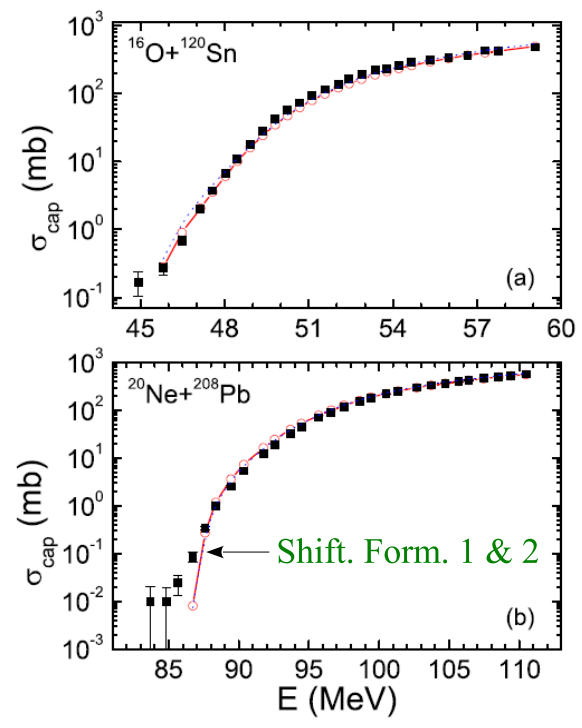

Figure 6. (Color online) The same as in Fig. 5, but for the capture cross sections. See Ref. [2] for details.

probabilities with the ratio (taken at 180 degrees) of the elastic and quasi-elastic cross sections with the Rutherford cross sections, respectively. In Fig. 4, CRC calculations for ${ }^{16} \mathrm{O}+{ }^{208} \mathrm{~Pb}$ at energies around the Coulomb barrier demonstrate that this idea works well, the breakup (BU) and deep-inelastic (DIC) components being negligible.

$$
\begin{aligned}
& J=\eta^{\prime} \cot \left[\frac{\theta}{2}\right] \\
& P_{e l}(\theta) \approx P_{e l}(J) \approx P_{e l}\left(\varepsilon_{J}, J=0\right)
\end{aligned}
$$
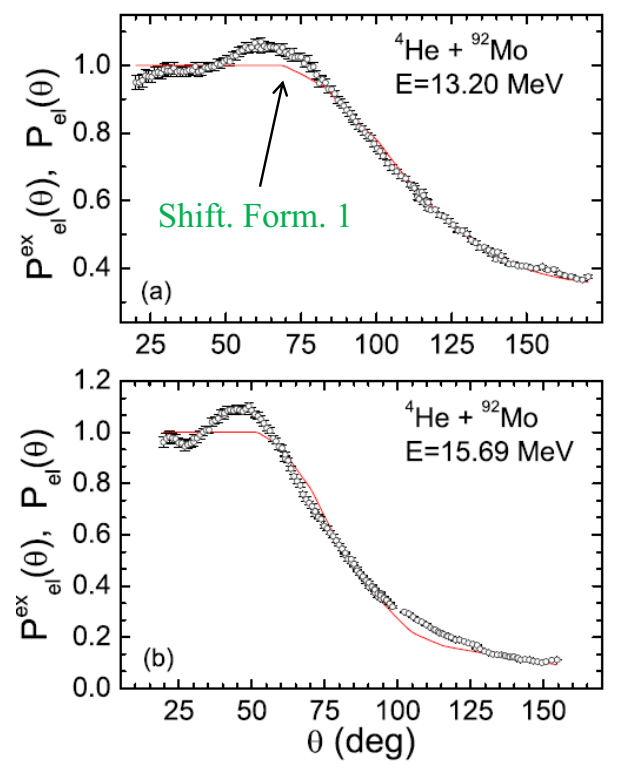

Figure 7. (Color online) Elastic-scattering probabilities from the simplified formulae compared with experimental data. See Ref. [2] for details.

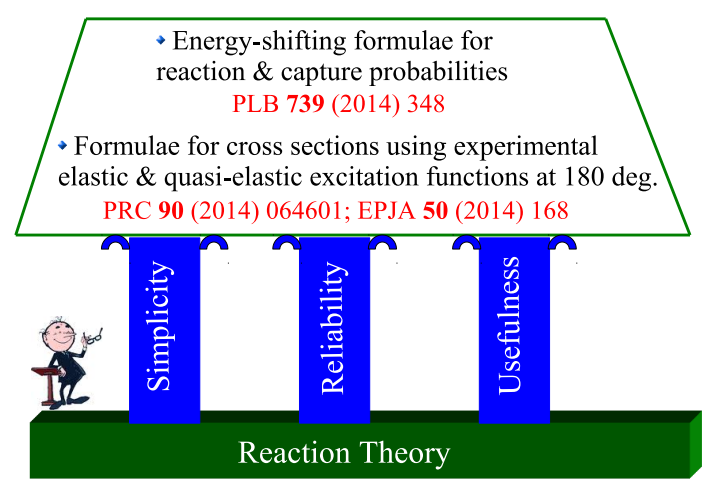

Figure 8. (Color online) A simplified, reliable and useful method, based on reaction theory, for predicting various cross sections.

As examples, Figs. 5 and 6 show predictions of the simplified formulae for the reaction and capture excitation functions of several heavy-ion collisions, respectively. It is observed that these predictions very well agree with experimental data. Using the semi-classical relationship between the scattering angle and the orbital angular momentum as well as the experimental elastic back-scattering excitation function, it is also possible to calculate elastic- 
scattering differential cross sections relative to the Rutherford cross sections (probabilities), as presented in Fig. 7. The agreement is in general very good, but (as expected) the formulae do not describe the data around the angular region of the Coulomb-nuclear interference peak as quantum coherence effects are not included.

\section{Summary}

Figure 8 encapsulates the key messages of the present contribution. Based on reaction theory and three pillars (simplicity, reliability and usefulness), we have presented a method for predicting a number of integrated and differential cross sections, using experimental information on elastic and quasi-elastic back-scattering excitation functions. For instance, this experimental information about elastic scattering allows one to calculate, through the present method, the reaction cross section. It seems to be simpler than the commonly used method based on measuring the full angular distribution of the elastic scattering.

\section{References}

[1] B.B. Back, H. Esbensen, C.L. Jiang, and K.E. Rehm, Rev. Mod. Phys. 86, 317 (2014)

[2] V.V. Sargsyan, G.G. Adamian, N.V. Antonenko, A. Diaz-Torres, P.R.S. Gomes and H. Lenske, Phys. Rev. C 90, 064601 (2014); Eur. Phys. J. A 50, 168 (2014)

[3] A. Diaz-Torres, G.G. Adamian, V.V. Sargsyan, and N.V. Antonenko, Phys. Lett. B 739, 348 (2014)

[4] I.J. Thompson, Comp. Phys. Rep. 7, 167 (1988)

[5] K. Hagino, N. Rowley, and A.T. Kruppa, Comp. Phys. Comm. 123, 143 (1999) 